\title{
Pre-dose Measure
}

National Cancer Institute

\section{Source}

National Cancer Institute. Pre-dose Measure. NCI Thesaurus. Code C106037.

A measure that relates specifically to the last value collected prior to the time of the first dose of a treatment. 